\title{
Calculation of MoM Interaction Integrals in Highly Conductive Media
}

\author{
Joris Peeters, Ignace Bogaert, and Daniël De Zutter Fellow IEEE \\ Department of Information Technology (INTEC), Ghent University, Sint-Pietersnieuwstraat 41, B-9000 Gent, \\ Belgium, e-mail: Joris.Peeters@intec.ugent.be
}

\begin{abstract}
The construction of the impedance matrix in the Method of Moments requires the calculation of interaction integrals between the expansion functions, through the Green's function and its derivatives. The singular behaviour of the Green's function poses considerable problems for an accurate numerical evaluation of these integrals, requiring techniques such as singularity extraction or cancellation. In this contribution we will show why these methods fail when the medium is highly conductive. A novel technique is proposed to handle these highly challenging integrals. The complexity of the new method is independent of the conductivity.
\end{abstract}

\section{INTRODUCTION}

The Method of Moments (MoM) is one of the most powerful approaches for solving electromagnetic scattering problems in piecewise homogeneous media. Its main advantage compared to other techniques, such as the Finite Difference Time Domain (FDTD) method and the Finite Elements (FE) method, is that only the surface of the objects must be discretised. The disadvantage, however, is that the resulting system matrix is fully dense, describing the interaction between all expansion functions by integrals with the singular 3D Green's function, given by $g(r)=\frac{e^{-j k r}}{4 \pi r}$, or its gradient as the kernel. Different techniques to calculate these integrals have been proposed in the past, focusing on regularising the $\frac{1}{r}$ behaviour. The two most prominent approaches are singularity extraction (SE) [1], [2], [3], [4] and singularity cancellation (SC) [5], [6], [7]. However, both these techniques assume that the numerator of the Green's function, i.e. $e^{-j k r}$, is a well-behaved function with a fairly small absolute value of the derivative. Indeed, for lossless media the wavenumber $k$ is real and for the usual $\frac{\lambda}{10}$ discretisation, the function $\cos \frac{2 \pi r}{\lambda}-j \sin \frac{2 \pi r}{\lambda}$ is smooth. In that case, the wavenumber $k$ is given by $\frac{\omega}{c} \sqrt{\epsilon_{r} \mu_{r}}$, with $\lambda=\frac{2 \pi}{k}$. For a very good conductor (with conductivity $\sigma \gg \omega \epsilon)$, we have that $k \approx \frac{1-j}{\delta}$, with the skin depth $\delta=\sqrt{\frac{2}{\omega \mu \sigma}}$ [8]. Note that, in general, as the conductivity $\sigma$ becomes larger, both the real part and imaginary part of $k$ grow, and are approximately equal to each other in magnitude. As a consequence, $e^{-j k r}$ becomes a function that is both highly oscillatory and exponentially damped and can by no means be considered as a smooth function to be handled by the standard numerical quadratures. In fact, as will be shown later in this contribution, a very specialised approach, tuned to this damped behaviour, is required in order to accurately evaluate the impedance integrals in highly conductive media. A similar topic has been treated in [9], but in a different manner that, to our knowledge, does not lead to a scalable solution (i.e. a calculation time that is independent of the conductivity $\sigma$, assuming the frequency does not vary). The outline of this paper is as follows. Section II introduces the MoM interaction integrals that occur when modelling a scattering problem at a body with complex $\epsilon$ and $\mu$. Section III gives a short overview of the currently most widely used techniques for calculating the singular or near singular impedance integrals and also explains the reason for their breakdown when the interacting medium is highly conductive. Section IV introduces our novel method for tackling the impedance integrals in these media and in Section V, this method is applied to a few challenging cases. Essentially, the full-wave treatment (as opposed to using a surface impedance) we propose is useful whenever the thickness of the conductor becomes of the order of or smaller than the skin depth. By means of numerical illustration and validation, Section VI applies our approach to the case of 'tunnelling' through a very thin conductive spherical shell. Additionally, some further fields of application are suggested that may benefit from this work.

\section{IMPEDANCE InTEGRALS IN THE MoM}

Discretisation of the Boundary Integral Equations (BIE) in the MoM leads to a dense linear system, the matrix elements of which describe the interaction, through the Green's function, between the expansion functions. Scattering at objects with a permittivity $\epsilon$ and permeability $\mu$ (but neither infinitely lossy) requires the introduction of two equivalent surface current densities, electric and magnetic, which can be solved for as the solution of the Poggio-Miller-Chang-Harrington-WuTsai (PMCHWT) [10] BIE. In this contribution, it will be assumed that the surface current densities are expanded into Rao-Wilton-Glisson (RWG) [11] functions (which we will denote as $\boldsymbol{b}(\boldsymbol{r})$ ), defined on a mesh of flat triangles, although the proposed techniques have a broader field of application (including an extension to a curvilinear mesh and the use of higher order basis functions). The resulting matrix elements require the calculation of the following integrals (as part of the $T$ and $K$ operators [2]) over the support $S_{i}$ of the test functions $\boldsymbol{b}_{i}$ and the support $S_{j}$ of basis functions $\boldsymbol{b}_{j}$ :

$$
\begin{gathered}
I_{1}=\int_{S_{i}} \boldsymbol{b}_{i}(\boldsymbol{r}) \cdot \int_{S_{j}} g(R) \boldsymbol{b}_{j}\left(\boldsymbol{r}^{\prime}\right) \mathrm{d} S^{\prime} \mathrm{d} S \\
I_{2}=\int_{S_{i}}\left[\nabla \cdot \boldsymbol{b}_{i}(\boldsymbol{r})\right] \int_{S_{j}} g(R)\left[\nabla^{\prime} \cdot \boldsymbol{b}_{j}\left(\boldsymbol{r}^{\prime}\right)\right] \mathrm{d} S^{\prime} \mathrm{d} S
\end{gathered}
$$




$$
I_{3}=\mathrm{PV} \int_{S_{i}} \boldsymbol{b}_{i}(\boldsymbol{r}) \cdot \int_{S_{j}} \nabla g(R) \times \boldsymbol{b}_{j}\left(\boldsymbol{r}^{\prime}\right) \mathrm{d} S^{\prime} \mathrm{d} S
$$

with $P V$ indicating the Principal Value of the integral. When the supports of $\boldsymbol{b}_{i}$ and $\boldsymbol{b}_{j}$ overlap in at least a point, the above integrals have a non-continuous integrand, although they are integrable. For $S_{i}=S_{j}$ (the self patch case), $I_{3}$ becomes zero. In order to determine these integrals over a triangle, for each possible $\boldsymbol{b}$, it suffices to calculate the following integrals:

$$
\begin{gathered}
I_{t 1}=\int_{S_{i}} \boldsymbol{r} \cdot \int_{S_{j}} g(R) \boldsymbol{r}^{\prime} \mathrm{d} S^{\prime} \mathrm{d} S \\
I_{t 2}=\int_{S_{i}} \int_{S_{j}} g(R) \mathrm{d} S^{\prime} \mathrm{d} S \\
\boldsymbol{I}_{t 3}=\mathrm{PV} \int_{S_{i}} \int_{S_{j}} \nabla g(R) \times \boldsymbol{r}^{\prime} \mathrm{d} S^{\prime} \mathrm{d} S
\end{gathered}
$$

To obtain the integrals $I_{1}, I_{2}$ and $I_{3}$, these integrals $I_{t 1}, I_{t 2}$ and $\boldsymbol{I}_{t 3}$ are required, in addition to some others that are merely variations in terms of the presence or absence of $\boldsymbol{r}$ or $\boldsymbol{r}^{\prime}$. The reason both $I_{t 1}$ and $I_{t 2}$ are included here, instead of just one of them, is to demonstrate in the examples that the presence of $\boldsymbol{r}$ has no mentionable influence on the achieveable accuracy. In short, if the three integrals above can be evaluated efficiently and accurately, this also guarantees accurate evaluation of all the integrals that are required in the impedance matrix.

In the next section, we will briefly revisit the techniques of Singularity Extraction and Singularity Cancellation, the workhorses behind most MoM implementations.

\section{CALCUlation OF IMPEDANCE INTEGRALS IN DIELECTRICS}

In order to obtain an accurate solution from the PMCHWT $\mathrm{BIE}$, it is essential that the integrals described in the previous section are evaluated with a relatively high accuracy. When the expansion functions $\boldsymbol{b}_{i}$ and $\boldsymbol{b}_{j}$ are well-separated (i.e. their distance from each other is considerably larger than their size), the integrand is sufficiently smooth and a straightforward Gaussian quadrature rule allows for exponential convergence. More challenging are the cases when the supports overlap (singular) or are very close (near-singular). Both situations require a specialised approach that deals with the singular or near-singular behaviour of the integrand.

We will first elaborate on the concept of Singularity Extraction, which is based on the fact that interaction integrals with static kernels, for example $\frac{1}{R}$, can be integrated analytically. As such, for instance, $I_{t 2}$ can be rewritten as:

$$
I_{t 2}=\left[\int_{S_{i}} \int_{S_{j}}\left[g(R)-\frac{1}{R}\right] \mathrm{d} S^{\prime} \mathrm{d} S+\int_{S_{i}} \int_{S_{j}} \frac{1}{R} \mathrm{~d} S^{\prime} \mathrm{d} S\right]
$$

The second double integral is evaluated analytically and the first double integral, from which the singular part is extracted, is now regular. Note that, even though the first integrand is now continuous, it is not $C^{\infty}$ because the first derivative, in this example, displays a discontinuity at $R=0$. Additional terms have to be extracted for continuity in the derivatives [2]. Singularity Extraction can also be applied to the near-singular case, in order to smooth the integrand and thus increasing the efficiency. An essential assumption behind the philosophy of Singularity Extraction is that, by extracting the singular (or near-singular) static part, the remaining integral automatically becomes suitable for numerical quadrature. As we will see later, in the case of conductive media, this is not the case.

A second technique, in competition with Singularity Extraction, is Singularity Cancellation. This method aims to regularise the integrand by a suitable change of coordinates. Considering again $I_{t 2}$ as an example, a simple yet effective transformation to polar coordinates in the inner integral would do the trick:

$$
I_{t 2}=\left[\int_{S_{i}} \mathrm{~d} S \int_{0}^{2 \pi} \mathrm{d} \phi \int_{0}^{R(\phi)} g(R) R \mathrm{~d} R\right]
$$

where we have assumed, in order to more clearly demonstrate the idea, that we are dealing with the self patch case $\left(S_{i}=\right.$ $\left.S_{j}\right)$. The Jacobian $(R)$ compensates the $\frac{1}{R}$ that appears in the Green's function and as such regularises the integrand to a $C^{\infty}$ function. An advantage of this approach is that it does not rely on the existence of analytical solutions for the static part. This allows for more flexibility in the expansion functions, paving the way for higher order solutions.

\section{CAlculation of IMPEdAnCE InTEGRALS IN Conductive Media}

In order to understand the difficulties that occur when calculating the impedance integrals in conductive media, it is instructive to look at the behaviour of the Green's function for various values of the conductivity $\sigma$, as shown in Fig. 1. The pulsation $\omega$ is chosen equal to $300 \mathrm{MHz}$. The distance $r$ is varied from 0 to $\frac{\lambda}{10}$, with $\lambda$ the free space wavelength (with $\left.\epsilon_{r}=\mu_{r}=1\right)$. Note how even a relatively poor conductor (with $\sigma=100 \mathrm{~S} \cdot \mathrm{m}^{-1}$ ) dampens the Green's function by more than five orders of magnitude over a distance of about $\frac{\lambda}{60}$. Copper, one of the most widespread conductors in industry, has $\sigma \approx 59.6 \cdot 10^{6} \mathrm{~S} \cdot \mathrm{m}^{-1}$, leading to a Green's function that is extremely localised around the origin.

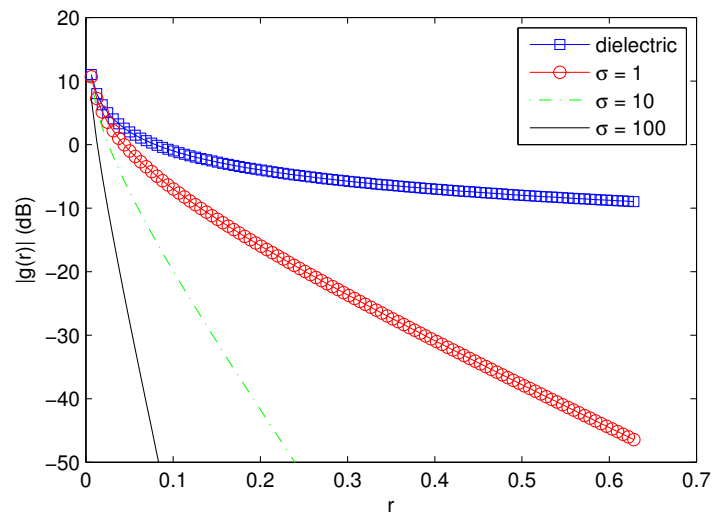

Figure 1. The absolute value of the Green's function $g(r)$ for a few values of $\sigma$.

This behaviour explains why straightforward application of techniques such as Singularity Extraction or Singularity 
Table I

THE WAVE NUMBER $k(1 / m)$ AND SKIN DEPTH $\delta(m)$ IN COPPER $\left(\sigma \approx 59.6 \cdot 10^{6} S \cdot m^{-1}\right)$ AS A FUNCTION OF THE FREQUENCY $f(\mathrm{~Hz})$ AND FREE SPACE WAVE NUMBER $k_{0}(1 / m)$.

\begin{tabular}{|c|c|c|c|}
\hline$f$ & $k_{0}$ & $k$ & $\delta$ \\
\hline $10^{3}$ & $2.96 \cdot 10^{-5}$ & $(4.8507-i 4.8507) \cdot 10^{2}$ & $2.06 \cdot 10^{-3}$ \\
$10^{6}$ & $2.96 \cdot 10^{-2}$ & $(1.5339-i 1.5339) \cdot 10^{4}$ & $6.52 \cdot 10^{-5}$ \\
$10^{9}$ & $2.96 \cdot 10^{1}$ & $(4.8507-i 4.8507) \cdot 10^{5}$ & $2.06 \cdot 10^{-6}$ \\
\hline
\end{tabular}

Cancellation break down for high conductivity, because they neglect the highly oscillatory but at the same time exponentially damped character of $e^{-j k r}$. In addition, SE suffers from numerical cancellation issues between the different extracted terms. A numerical comparison for conductive media between $\mathrm{SE}, \mathrm{SC}$ and our novel approach will be given further in this paper.

In order to introduce our new technique for treating these integrals in conductive media, the explanation will be based on $I_{t 2}$. Further on it will also be shown how both $I_{t 1}$ and $I_{t 3}$ can be treated almost identically. So, in the remainder of this section, we will be looking at a way to efficiently evaluate the following integral:

$$
I_{i j}=\int_{S_{i}} \int_{S_{j}} g(R) \mathrm{d} S^{\prime} \mathrm{d} S
$$

for arbitrary values of $\sigma$. In order to do this, a specialised approach is required for both the inner and outer integrals.

\section{A. Inner Integral}

First, we will take a look at evaluating the inner integral, namely

$$
I_{j}(\boldsymbol{r})=\int_{S_{j}} g\left(\left|\boldsymbol{r}-\boldsymbol{r}^{\prime}\right|\right) \mathrm{d} S^{\prime}
$$

where, although $r$ can be anywhere in space, the most challenging and practically interesting cases are when $r$ is very close to $S_{j}$ or even in it. The key to accurately integrate the strongly pulsed behaviour is focusing the numerical quadrature points only in those regions where the Green's function has a non-negligible value, based on a certain tolerance $\varepsilon$. The wave number in a good conductor approximately satisfies

$$
k \approx \frac{|k|}{\sqrt{2}}(1-j)
$$

with $|k| \approx \sqrt{\omega \mu \sigma}$ (see Table I for some numerical values using copper as an example). This allows us to approximately express the numerator of the Green's function in terms of $|k r|$ only,

$$
e^{-j k r} \approx\left[\cos \frac{|k r|}{\sqrt{2}}-j \sin \frac{|k r|}{\sqrt{2}}\right] e^{-\frac{|k r|}{\sqrt{2}}}
$$

The behaviour of this function is illustrated in Fig. 2 (with $x=|k r|)$.

Beyond a certain electrical length, the numerator of the Green's function drops to a fraction $\varepsilon_{c u t}$ compared to its value in the origin. As such a certain cut-off value of $r$ can be determined, beyond which the remainder can be neglected, namely

$$
r_{c u t}=-\frac{\sqrt{2} \ln \varepsilon_{c u t}}{|k|}=-\delta \ln \varepsilon_{c u t}
$$

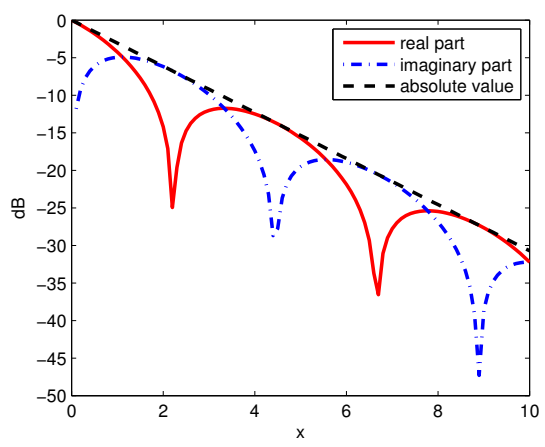

Figure 2. The real part, imaginary part and absolute value of the function $\left[\cos \frac{x}{\sqrt{2}}-j \sin \frac{x}{\sqrt{2}}\right] e^{-\frac{x}{\sqrt{2}}}$.

With this knowledge, the inner integral can now be evaluated to any desired tolerance independent of $\sigma$. As a first step, a similar transform as in the Singularity Cancellation method is employed, namely a Duffy transformation, see, e.g. [3]. As mentioned before, this allows for more flexibility in the integrand and will in fact allow us to treat the inner integrals of $I_{t 1}, I_{t 2}$ and $I_{t 3}$ in an identical manner, despite the different kernel. With respect to a carefully selected $\boldsymbol{r}_{o}$ the integral $I_{j}(\boldsymbol{r})$ is transformed to polar coordinates $(\rho, \phi)$ :

$$
I_{j}(\boldsymbol{r})=\int_{\phi_{1}}^{\phi_{2}} \mathrm{~d} \phi \int_{\rho_{1}(\phi)}^{\rho_{2}(\phi)} g\left(\left|\boldsymbol{r}-\left(\boldsymbol{r}_{0}+\rho \boldsymbol{u}_{\rho}\right)\right|\right) \rho \mathrm{d} \rho
$$

This point $\boldsymbol{r}_{o}$ is found by first projecting $\boldsymbol{r}$ into the plane of the triangle $S_{j}$ and calling this projection $\boldsymbol{r}_{p}$. If $\boldsymbol{r}_{p}$ lies within $S_{j}$, it is equal to $\boldsymbol{r}_{o}$. If $\boldsymbol{r}_{p}$ lies outside the triangle, $\boldsymbol{r}_{o}$ is that point on the edge of the triangle that lies closest to $\boldsymbol{r}_{p}$. This process of finding $\boldsymbol{r}_{o}$ is illustrated in Fig. 3 .

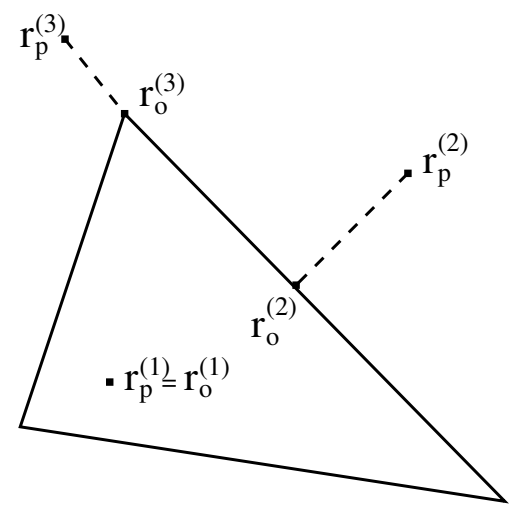

Figure 3. The point $\boldsymbol{r}_{o}$ is found as the point on the triangle (or its edge) that is closest to $\boldsymbol{r}_{p}$. This is illustrated for three different possibilities of $\boldsymbol{r}_{p}$.

Once $\boldsymbol{r}_{o}$ is determined, $S_{j}$ is divided into one, two or three triangles (depending on the location of $\boldsymbol{r}_{o}$ ), each having $\boldsymbol{r}_{o}$ as one of their corners. This is illustrated in Fig. 4. The total integral is expressed as the sum of the integrals over these subtriangles, a similar approach as, e.g., [7] and [12].

The integration over one subtriangle can be rewritten as

$$
I_{j}(\boldsymbol{r})=\int_{0}^{\phi_{e}} \mathrm{~d} \phi \int_{0}^{\rho_{e}(\phi)} g\left(\left|\boldsymbol{r}-\left(\boldsymbol{r}_{0}+\rho \boldsymbol{u}_{\rho}\right)\right|\right) \rho \mathrm{d} \rho
$$




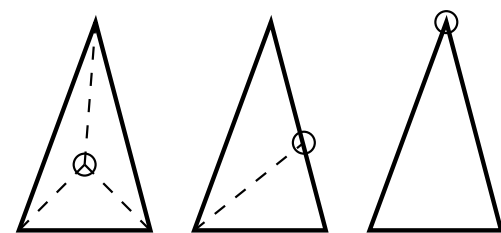

Figure 4. The division into subtriangles for three different cases. The location of $\boldsymbol{r}_{o}$ is indicated by the small circle. Left: $\boldsymbol{r}_{o}$ lies in the triangle, which is subdivided into three parts. Middle: $\boldsymbol{r}_{o}$ lies on the edge of the triangle, which is subdivided into two parts. Right: $\boldsymbol{r}_{o}$ lies on the corner of the triangle and no subdivision is needed.

Let us first look at the radial integration for a subtriangle:

$$
I_{j}(\boldsymbol{r}, \phi)=\int_{0}^{\rho_{e}(\phi)} g\left(\left|\boldsymbol{r}-\left(\boldsymbol{r}_{0}+\rho \boldsymbol{u}_{\rho}\right)\right|\right) \rho \mathrm{d} \rho
$$

The endpoint of the integration $\rho_{e}(\phi)$ is dependent on the triangle shape, on the angular coordinate and also on the value of $r_{c u t}$, which might truncate the integration domain. The latter occurs when the endpoint is further away from $r$ than the distance $r_{\text {cut }}$. In that case, the integration is carried out from $\rho=0$ to $\rho=\rho_{e, t}$, such that the new endpoint is $r_{c u t}$ away from $r$. This is illustrated in Fig. 5. Clearly, this cut-off does not compromise the accuracy, due to the rapid decay of the Green's function.

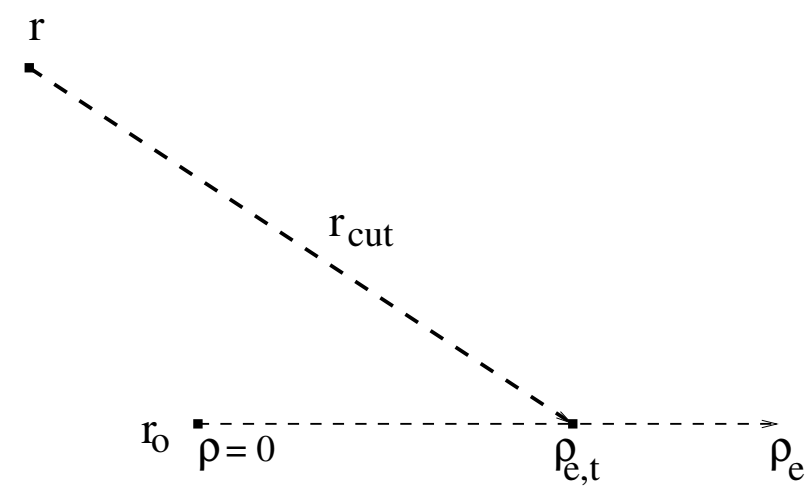

Figure 5. The truncation of the radial integration domain from $\left[0, \rho_{e}\right]$ to $\left[0, \rho_{e, t}\right]$ to keep all quadrature points within a distance $r_{c u t}$ from $\boldsymbol{r}$.

Regarding the shape of the integrand, it must be noted that, while the polar coordinate transform is capable of cancelling out a $\frac{1}{R}$ singularity, $I_{3 j}$ leads to a $\frac{1}{R^{2}}$ singularity. In addition, due to the conductive behaviour, the function will in any case have its least smooth behaviour around $\rho=0$, although the limitation of radial distance to $r_{c u t}$ largely solves this problem. One interesting approach to tackle integrands such as $I_{3 j}$ with possible endpoint singularities is the Double Exponential (DE) transform [13], essentially mapping a $[-1,1]$ region on a $[-\infty,+\infty]$ region that can be handled with a trapezoidal rule to exponential accuracy. This allows for the desired flexibility in terms of kernel and expansion functions. The radial integrand now becomes (supressing dependencies of $\phi$ and assuming $\rho_{e}$ to indicate the integration endpoint, whether or not truncated to $\left.\rho_{e, t}\right)$ :

$$
I_{j}(\boldsymbol{r}, \phi)=\frac{\rho_{e}}{2} \int_{-\infty}^{\infty} g\left(\left|\boldsymbol{r}-\left(\boldsymbol{r}_{0}+\rho(t) \boldsymbol{u}_{\rho(t)}\right)\right|\right) \rho(t) \psi^{\prime}(t) \mathrm{d} t
$$

in which $\rho(t)=\frac{\rho_{e}}{2} \psi(t)+\frac{\rho_{e}}{2}$ and with $\psi(t)$ the so-called double exponential transform given by $\psi(t)=\tanh \left(\frac{\pi}{2} \sinh (t)\right)$. To our knowledge, the DE transform was first employed for the calculation of impedance integrals in [12], which also contains a large amount of background on the technique. The essential difference with regard to conductive integrals is the use of $r_{c u t}$ in this work. An alternative for DE is using Gauss quadrature. Even though it cannot handle the singular behaviour of $I_{3 j}$, it performs better for those integrals that are regularised by the Duffy Transform (i.e. achieves roughly one or two orders of magnitude additional precision for the same number of quadrature points). So, in the case of the self-patch, when $I_{3 j}$ is zero, it would lead to a more efficient solution. However, in any case different from the self-patch we would need the K-operator in addition to the T-operator, so our recommendation is to use the DE transform to calculate the different radial integrals simultaneously, which reduces the number of evaluations of the Green's function, whilst still achieving any practically desired tolerance. So, in the remainder of this article we will use the DE transform (like in our own MoM implementation for these integrals), but the reader should be aware that Gauss quadrature can be a decent alternative in some cases, but unfortunately fails in others. As an example, integral (17) is evaluated for the following data: $\boldsymbol{r}=(0,0, d), \boldsymbol{r}_{o}=(0,0,0), \boldsymbol{u}_{\rho}=(1,0,0)$ and $\rho_{e}=1$. The results are given in Table II for a few choices of the parameters. The use of the truncation distance $r_{c u t}$ essentially imposes a maximal absolute error on the integral. If the interaction distance $d$ is well beyond the skin depth $\delta$, this may lead to a large relative error (because the value of the integral is very small). However, in the MoM scheme, it is pointless to evaluate these integrals to higher precision because they barely contribute. Essentially, the more distant an interaction, the less accurate its evaluation needs to be. That is exactly what the use of $r_{\text {cut }}$ accomplishes. Note that in all numerical experiments, both here and in the next sections, the values of $\epsilon_{r e l}$ are obtained through comparison with a numerical result using a much higher amount of quadrature points, which is used as the reference result.

Having control over the radial integral, it is now used as the integrand for the angular quadrature:

$$
I_{j}(\boldsymbol{r})=\int_{0}^{\phi_{e}} I_{j}(\boldsymbol{r}, \phi) \mathrm{d} \phi
$$

Regarding the choice of quadrature rule and number of sample points needed to evaluate (18), it is important to notice that, when $r_{c u t}$ is small compared to the dimensions of the triangle, the integrand is actually not strongly dependent on $\phi$ because in that case only a limited portion of the triangle has to be integrated over. This is illustrated in Fig. 6. As such, in those cases, as little as one integration point is usually sufficient. When the conductivity is high, these cases will occur quite often and it is worth detecting them. If the complete triangle plays a role, then a Gaussian quadrature rule is employed. For 
Table II

THE RELATIVE ERROR $\left(\varepsilon_{r e l}\right)$ AND ABSOLUTE ERROR $\left(\varepsilon_{a b s}\right)$ FOR THE NUMERICAL EVALUATION OF THE RADIAL INTEGRAL (17). THE NUMBER OF QUADRATURE POINTS FOR THE DOUBLE EXPONENTIAL FORMULA IS $n_{\rho}$

\begin{tabular}{|c|c|c|c|c|c|}
\hline$\delta$ & $\frac{d}{\delta}$ & $\varepsilon_{c u t}$ & $n_{\rho}$ & $\varepsilon_{\text {rel }}$ & $\varepsilon_{a b s}$ \\
\hline $10^{-2}$ & $10^{1}$ & $10^{-3}$ & 17 & 1 & $1.8 \cdot 10^{-8}$ \\
$10^{-2}$ & $10^{1}$ & $10^{-5}$ & 17 & 0.6 & $1.07 \cdot 10^{-9}$ \\
$10^{-2}$ & $10^{1}$ & $10^{-7}$ & 17 & 0.11 & $2.05 \cdot 10^{-10}$ \\
$10^{-2}$ & $10^{1}$ & $10^{-7}$ & 33 & $1.5 \cdot 10^{-4}$ & $2.77 \cdot 10^{-12}$ \\
$10^{-2}$ & $10^{1}$ & $10^{-7}$ & 65 & $2.8 \cdot 10^{-5}$ & $5.05 \cdot 10^{-13}$ \\
$10^{-5}$ & $10^{-10}$ & $10^{-3}$ & 17 & $1.2 \cdot 10^{-2}$ & $4.92 \cdot 10^{-8}$ \\
$10^{-5}$ & $10^{-10}$ & $10^{-3}$ & 33 & $5.8 \cdot 10^{-5}$ & $2.3 \cdot 10^{-10}$ \\
$10^{-5}$ & $10^{-10}$ & $10^{-3}$ & 65 & $5.8 \cdot 10^{-5}$ & $2.3 \cdot 10^{-10}$ \\
$10^{-5}$ & $10^{-10}$ & $10^{-5}$ & 33 & $1.8 \cdot 10^{-5}$ & $7.1 \cdot 10^{-11}$ \\
$10^{-5}$ & $10^{-10}$ & $10^{-5}$ & 65 & $8.5 \cdot 10^{-8}$ & $3.4 \cdot 10^{-13}$ \\
$10^{-5}$ & $10^{-10}$ & $10^{-7}$ & 65 & $1.3 \cdot 10^{-10}$ & $5 \cdot 10^{-16}$ \\
\hline
\end{tabular}

most practical purposes, 8 sample points in $\phi$ turn out to be sufficient. In order to illustrate the obtainable accuracy and the fact that the complexity is independent of the conductivity, we consider the following example. The triangle is defined by the vertices $(0,0,0),(1,0,0)$ and $(0,1,0)$, the first of which is chosen to be $\boldsymbol{r}_{o}$. Table III shows the accuracy of the angular integral for a few locations of the observer point $r$, a few values of $k$ and different numbers $n_{\phi}$ of sample points for the angular integration. The radial integration was performed with sufficient accuracy so as not to influence the results.

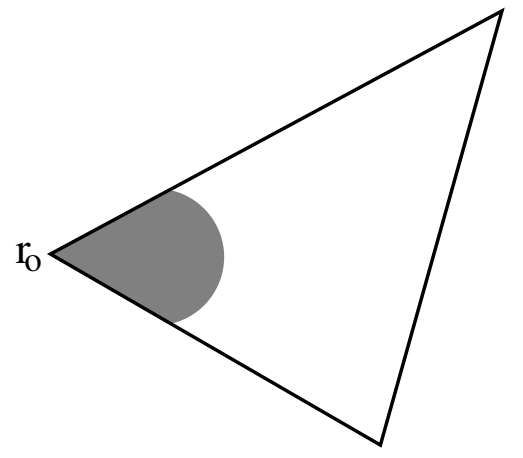

Figure 6. The integration domain when $r_{c u t}$ is smaller than the dimensions of the triangle. In contrast, when $r_{c u t}$ becomes larger, the integration domain becomes the entire triangle.

Table III

THE RELATIVE ERROR $\left(\varepsilon_{r e l}\right)$ AND ABSOLUTE ERROR $\left(\varepsilon_{a b s}\right)$ FOR THE NUMERICAL EVALUATION OF THE ANGULAR INTEGRAL (18).

\begin{tabular}{|c|c|c|c|c|c|}
\hline$\delta$ & $r_{\text {cut }}$ & $\boldsymbol{r}$ & $n_{\phi}$ & $\varepsilon_{r e l}$ & $\varepsilon_{a b s}$ \\
\hline $10^{-3}$ & 0.011 & $(0.0,0.0,0.0)$ & 1 & $9.99 \cdot 10^{-6}$ & $4.4 \cdot 10^{-11}$ \\
$10^{-3}$ & 0.018 & $(0.0,0.0,0.0)$ & 1 & $9.99 \cdot 10^{-6}$ & $4.4 \cdot 10^{-10}$ \\
$10^{-2}$ & 0.11 & $(0.0,0.0,0.0)$ & 1 & $9.99 \cdot 10^{-6}$ & $4.4 \cdot 10^{-9}$ \\
$10^{-2}$ & 0.11 & $(0.0,0.0,0.1)$ & 1 & 0.22 & $4.4 \cdot 10^{-9}$ \\
$10^{-2}$ & 0.11 & $(0.0,0.0,0.2)$ & 1 & 1 & $4.4 \cdot 10^{-13}$ \\
$10^{-1}$ & 1.15 & $(0.0,0.0,0.2)$ & 1 & $8.18 \cdot 10^{-5}$ & $4.8 \cdot 10^{-8}$ \\
$10^{-1}$ & 1.15 & $(0.0,0.0,0.2)$ & 8 & $2.49 \cdot 10^{-5}$ & $15 \cdot 10^{-8}$ \\
$10^{-1}$ & 1.15 & $(0.0,0.0,0.2)$ & 16 & $2.34 \cdot 10^{-5}$ & $1.4 \cdot 10^{-8}$ \\
\hline
\end{tabular}

It is clear that the previously described methods allow for efficient and accurate evaluation of the inner integral (14). Application of the DE technique and the introduction of $r_{\text {cut }}$ makes the calculation time and accuracy independent of
Table IV

THE RELATIVE ERRORS AS A FUNCTION OF $k=q-i q$ FOR EVALUATION OF THE INNER INTEGRAL USING SINGULARITY EXTRACTION (WITH 15 TERMS), SINGULARITY CANCELLATION (WITH 17 QUADRATURE POINTS BOTH FOR THE RADIAL PART AND THE ANGULAR PART) AND OUR NOVEL APPROACH (USING THE SAME AMOUNT OF QUADRATURE POINTS AS SC AND $r_{c u t}$ FOR A TOLERANCE OF $10^{-2}$ ).

\begin{tabular}{|c|c|c|c|}
\hline$q$ & $\epsilon_{\text {rel }, \text { SE }}$ & $\epsilon_{\text {rel,SC }}$ & $\epsilon_{\text {rel, novel }}$ \\
\hline $10^{0}$ & $3 \cdot 10^{-7}$ & $2 \cdot 10^{-7}$ & $2 \cdot 10^{-7}$ \\
$10^{1}$ & $1 \cdot 10^{-6}$ & $3 \cdot 10^{-7}$ & $5 \cdot 10^{-4}$ \\
$10^{2}$ & $3 \cdot 10^{29}$ & $5 \cdot 10^{-5}$ & $1 \cdot 10^{-2}$ \\
$10^{3}$ & $4 \cdot 10^{69}$ & $8 \cdot 10^{-1}$ & $1 \cdot 10^{-2}$ \\
$10^{4}$ & $4 \cdot 10^{109}$ & $1 \cdot 10^{0}$ & $1 \cdot 10^{-2}$ \\
\hline
\end{tabular}

the conductivity. The inner integral will now serve as the integrand of the outer integral, over triangle $S_{i}$. However, before moving on to the outer integral, the numerical accuracy and efficiency of our treatment of the inner integral will be compared with that of Singularity Extraction and Singularity Cancellation. The latter comes in many shapes, but here we will simply employ our previously discussed technique, but setting $r_{\text {cut }}=\infty$, which reduces to a typical Duffy Transform. Of course, other cancellation approaches may lead to different results, but this example merely serves as an illustration of the problems that will occur in, to our belief, all of them. For Singularity Extraction, we use a formulation without a 'regular remainder', i.e. we extract as many analytical terms as is necessary for a sufficiently accurate result (in this case 15 terms) if there was no conductivity. For the numerical example, we take a source triangle that has vertices at $(0,0,0),(1,0,0)$ and $(0,1,0)$, with an observer point $10^{-7}$ above its center of mass and we use a wavenumber $k$ of the form $q-i q$. The results are shown in Table IV.

As can be observed, the Singularity Extraction method becomes numerically unstable and diverges, due to the many terms that suffer from numerical cancellation. If only one or two terms are extracted (instead of 15) and the remainder is integrated numerically, a similar problem as with the Singularity Cancellation technique will appear, which can not keep up with the increasingly rapid variation of the Green's function and loses accuracy. The novel approach is less accurate and more expensive at low losses but manages to stay within the chosen tolerance for the cases of high conductivity, whereas other methods fail in this region.

\section{B. Outer Integral}

The outer integral is given by

$$
I_{i j}=\int_{S_{i}} I_{j}(\boldsymbol{r}) \mathrm{d} S
$$

and the others have a similar form and can also be treated in a completely identical manner as will be described in this subsection. However, for the sake of the argument, the approach will be focusing on $I_{i j, 2}$. For the inner integral, the key to efficient evaluation was a focusing of quadrature points in the regions where the integrand is non-negligible (through the choice of $r_{c u t}$ and the use of the DE transform). A similar objective lies behind the philosophy of the proposed method to evaluate the outer integral. As an example, and to illustrate the 
difficulties, Fig. 7 shows two triangles $S_{i}$ and $S_{j}$ that, when projected onto each other, overlap only partially. When the conductivity is high, the parts on $S_{i}$ that are not very close to $S_{j}$ (basically within the $r_{c u t}$ range as previously determined) will hardly contribute to the outer integral. If the two triangles are parallel and right above each other, the integrand will in fact hardly change at all. The only regions on $S_{i}$ where the outer integrand is not smooth are those that are very close to an edge of $S_{j}$, because in that case the inner integrand and hence the result of the inner integration changes rapidly. In order to accurately evaluate the integral, these latter regions will require special care. Our novel approach is designed to determine those parts of $S_{j}$ that contribute to the outer integral and to focus the quadrature points in those regions where the integrand changes rapidly.

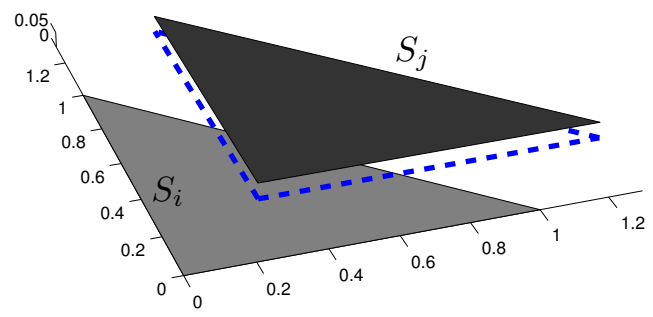

Figure 7. The situation of two triangles that only partially overlap when projected onto each other, with the dashed line indicating the projection of the top triangle on the plane of the bottom triangle.

As a first step, the integration region on $S_{i}$ is reduced by eliminating those parts that are too far from the plane of $S_{j}$ to give any contribution. This is obtained by calculating the intersection (if any) between $S_{i}$ and the region between two planes, one at a distance $r_{c u t}$ below and parallel to $S_{j}$ and a similar plane above $S_{j}$. Depending on the configuration, this leads to a single polygon with three, four or five edges. If there is no intersection, $S_{i}$ is too far away from $S_{j}$ and the entire interaction integral, in view of the previously chosen tolerance $\varepsilon$, can be considered zero. This process is illustrated in Fig. 8.

The next step attempts to further reduce the integration domain and also identifies those regions where a rapid change of the integrand can be expected. This in turn leads to a subdivision of the integration domain in judiciously chosen subtriangles, such that in the end quadrature points are distributed in such a way that the overall integration precision is guaranteed. In order to achieve this, $S_{j}$ is first projected onto the plane of $S_{i}$. This projection is subsequently extended (in the plane of $S_{i}$ ) with polygons, covering a distance of at least $r_{c u t}$ from the original projection. The reduced integration domain for the outer integral is then determined as the intersection between $S_{i}$ and $S_{j}$ 's projection including its extensions. This process is illustrated in Fig. 9. In this particular example, the plane of

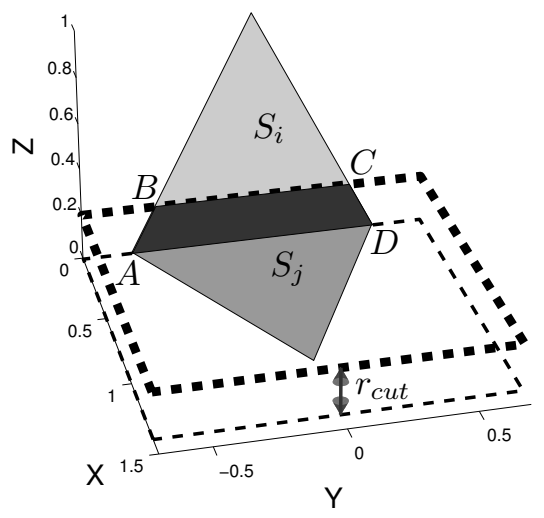

Figure 8. The domain of $S_{i}$ (lying in the yz-plane) is reduced to the darkly shaded area (the polygon ABCD), which is the intersection between $S_{i}$ and the (infinite) volume described by the plane of $S_{j}$ (dashed thin line in the xy-plane) and its upward projection (dashed thick line) over a distance $r_{c u t}$.

triangle $S_{j}$ is parallel to that of $S_{i}$ (the geometry is shown in Fig. 7). The solid black line in Fig. 9.a represents the projection $S_{j, p}$ of $S_{j}$ in the plane of $S_{i}$. We now first extend this projection $S_{j, p}$ over a distance $r_{c u t}$ to the outside. This extension is also shown in Fig. 9.a (the dashed lines). From this it follows that the integration over $S_{i}$ can be restricted to the darkly shaded area (denoted $\hat{S}$ ). Refering to the reasoning put forward w.r.t. the integration over $\phi$ in (18), it is clear that the integrand will not vary uniformly over $\hat{S}$. In order to guarantee the overall integration precision, the boundary of the projected triangle $S_{j, p}$ is now also extended to the inside as depicted in Fig. 9.b, finally leading to the subdivision of $\hat{S}$ in elementary integration polygons (6 in this particular example), as shown in Fig. 9.c. The numerical integration over these polygons now leads to an overall positioning of the sample points accounting for the exponential variation of the integrand imposed by the Green's functions (see Fig. 10 for a detail of the behaviour of this integrand), as such making sure that the precision obtained for the inner integration (10) does not get compromised when performing the outer integration (19).

To further illustrate the principles put forward by means of the example of Fig. 9, we again turn to the example shown in Fig. 8. In this special case, the projection $S_{j, p}$ of $S_{j}$ on $S_{i}$ reduces to the line $A D$ in Fig. 8, as the planes of $S_{i}$ and $S_{j}$ are perpendicular. Applying the procedure followed in the example of Fig. 9 now simply amounts to the reduction of the outer integration domain to the polynomial $A B C D$.

The combination of the first and second steps guarantees that the integrand in each polygon is non-negligible and that each possible steep variation is covered by one polygon. In a final step the actual integration needs to be carried out over these domains. The easiest approach, which delivers accurate results, is to divide each polyon into triangles and then consider all these triangles separately. Numerical quadrature over a triangle is already present in most implementations, reducing the amount of programming required. 


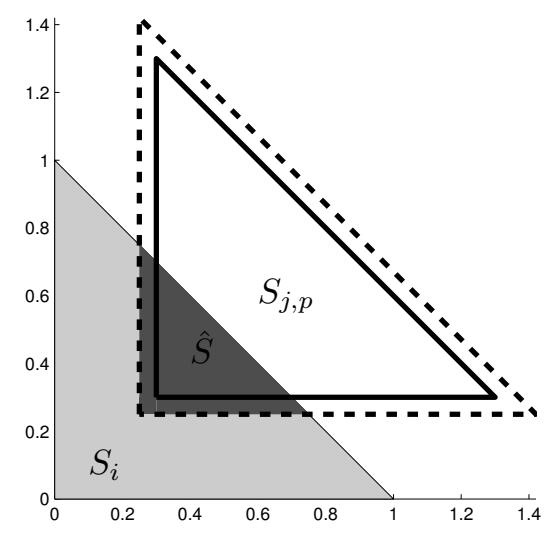

(a)

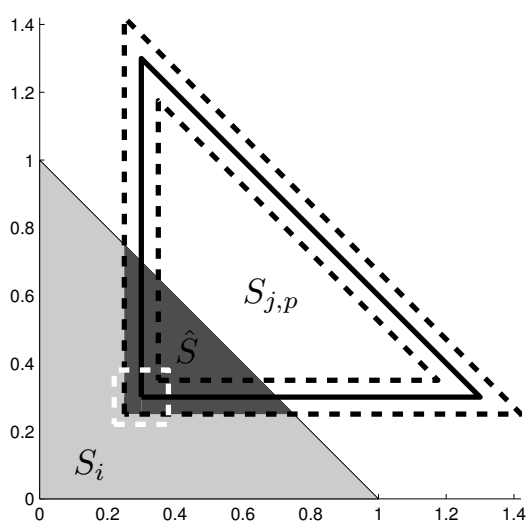

(b)

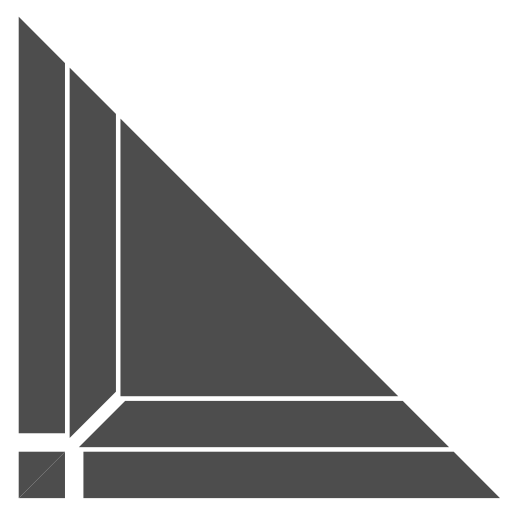

(c)

Figure 9. (a) Triangle $S_{i}$ (lightly and darkly shaded area) is reduced to the region forming its intersection with the projection $S_{j, p}$ (solid line) of triangle $S_{j}$ and its extensions to the outside over a distance $r_{c u t}$ (dashed lines). The darkly shaded area $\hat{S}$ is the resulting domain for the outer integration. (b) In addition to the outward extension, the projection $S_{j, p}$ must also be extended inwards. This does not change the total integration domain, but it influences the division into polygons. The white dashed lines indicate the region that is shown in detail in Fig. 10 (c) Schematic representation of the total integration domain (identical to the darkly shaded area in (a) and (b)), subdivided into the polygons over which the individual integrations takes place.

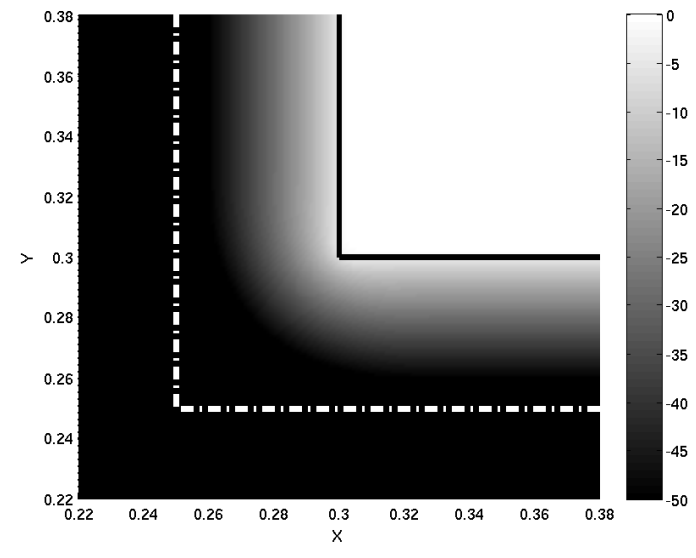

(a)

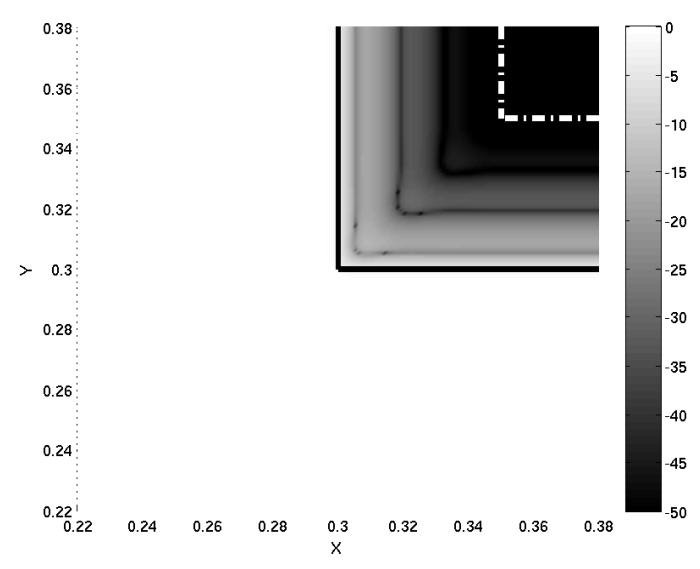

(b)

Figure 10. (a) A detail of the absolute value of the inner integrand (in $\mathrm{dB}$ ), in the region indicated by the white dashed lines in Fig. 9.b. In that example, $r_{c u t}$ is equal to 0.05 and was chosen to achieve a $10^{-5}$ accuracy. Here we see the exponential behaviour of the integrand near the edges (indicated by the black lines), dropping below $10^{-5}$ beyond a distance $r_{c u t}$. The outward extension of the projection is indicated by the white dashdot line. (b) Similar to (a), but now showing the absolute value of the inner integrand (in $\mathrm{dB}$ ) minus its value at $(0.4,0.4,0)$. The white dashdot lines indicate the inward extension.

\section{PERFormanCE}

This section will evaluate the performance and accuracy for calculating the impedance integrals for a few of the most interesting and challenging cases. The techniques described in the previous sections will be applied to each of the integrals $I_{t 1}, I_{t 2}$ and $\boldsymbol{I}_{t 3}$. Three particular geometrical situations will be considered that are of particular importance to potential applications. These are the so-called self patch (when two triangles overlap), the orthogonal neighbour patch (when they touch in a line and have orthogonal planes) and the case of two parallel triangles that are close to each other. Note that the self patch for $I_{3}$ is always zero and consequently that the self patch for $\boldsymbol{I}_{t 3}$ does not need to be calculated. Note that the accuracy of all results has been obtained through self-convergence (using the same method but with higher precision and, consequently, more quadrature points). For the low conductivity cases, our 
technique for the inner integral has been compared with SE and SC (see also Table IV), which verifies the implementation. The evaluation of the outer integral has been compared with an adaptive technique (progressive refinement of the integration region into more triangles), which is incredibly slow for higher conductivity but does, eventually, confirm our results. For every result, we generated a reference value that is at least two orders of magnitude more precise, in terms of all parameters (number of quadrature points for the radial, angular and outer integral, as well as the tolerance for $r_{c u t}$ ).

\section{A. Self Patch}

The first example under consideration is that of the interaction between two identical triangles, which is the cornerstone of the impedance matrix. The triangle is defined by the vertices $(0,0,0),(1,0,0)$ and $(0,1,0)$. The material through which they interact is chosen to be copper $\left(\sigma \approx 59.6 \cdot 10^{6} \mathrm{~S} \cdot \mathrm{m}^{-1}\right)$ and the self patch integral is studied at different frequencies. The challenging situations are those for which $\delta$ is small (or, equivalently, $|k|$ is large), which happens in the limits of high conductivity and high frequency. The results are shown in Table V. Note that the self patch contribution to the K-operator is always zero [10], hence the omission of $\boldsymbol{I}_{t 3}$ for this example.

Table V

THE RELATIVE ERRORS $\left(\varepsilon_{r e l, 1}\right.$ AND $\left.\varepsilon_{r e l, 2}\right)$ FOR THE NUMERICAL EVALUATION OF THE IMPEDANCE INTEGRALS $I_{t 1}$ AND $I_{t 2}$ IN THE CASE OF A SELF PATCH. THE NUMBER OF QUADRATURE POINTS USED FOR THE OUTER INTEGRATION (19) IS GIVEN BY $n_{O}$

\begin{tabular}{|c|c|c|c|c|c|}
\hline $\mathrm{f}(\mathrm{Hz})$ & $\delta$ & $\varepsilon_{c u t}$ & $\left(n_{O}, n_{\phi}, n_{\rho}\right)$ & $\varepsilon_{r e l, 1}$ & $\varepsilon_{\text {rel }, 2}$ \\
\hline $10^{4}$ & $6.4 \cdot 10^{-4}$ & $10^{-3}$ & $(24,4,33)$ & $1.2 \cdot 10^{-3}$ & $1.1 \cdot 10^{-3}$ \\
$10^{4}$ & $6.4 \cdot 10^{-4}$ & $10^{-5}$ & $(312,8,33)$ & $1.9 \cdot 10^{-5}$ & $1.1 \cdot 10^{-5}$ \\
$10^{5}$ & $2.0 \cdot 10^{-4}$ & $10^{-3}$ & $(24,4,33)$ & $1.0 \cdot 10^{-3}$ & $0.9 \cdot 10^{-3}$ \\
$10^{5}$ & $2.0 \cdot 10^{-4}$ & $10^{-5}$ & $(144,4,33)$ & $1.2 \cdot 10^{-6}$ & $5.1 \cdot 10^{-6}$ \\
$10^{6}$ & $6.4 \cdot 10^{-5}$ & $10^{-3}$ & $(24,4,33)$ & $1.0 \cdot 10^{-3}$ & $1.0 \cdot 10^{-3}$ \\
$10^{6}$ & $6.4 \cdot 10^{-5}$ & $10^{-5}$ & $(84,4,33)$ & $3.2 \cdot 10^{-6}$ & $3.8 \cdot 10^{-6}$ \\
$10^{7}$ & $2.0 \cdot 10^{-5}$ & $10^{-3}$ & $(24,4,33)$ & $1.0 \cdot 10^{-3}$ & $1.0 \cdot 10^{-3}$ \\
$10^{7}$ & $2.0 \cdot 10^{-5}$ & $10^{-5}$ & $(84,4,33)$ & $7.0 \cdot 10^{-6}$ & $8.0 \cdot 10^{-6}$ \\
$10^{8}$ & $6.4 \cdot 10^{-6}$ & $10^{-3}$ & $(24,4,33)$ & $1.0 \cdot 10^{-3}$ & $1.0 \cdot 10^{-3}$ \\
$10^{8}$ & $6.4 \cdot 10^{-6}$ & $10^{-5}$ & $(84,4,33)$ & $1.0 \cdot 10^{-5}$ & $0.9 \cdot 10^{-5}$ \\
\hline
\end{tabular}

The results show that our approach is stable for small $\delta$ and can also achieve a desired tolerance, for the frequency ranging over many orders of magnitude. Further numerical tests show that our approach is stable for $\delta$ at least as small as $10^{-14} \mathrm{~m}$, indicating the inherent robustness of our approach. Actually, the critical parameter in determining the behaviour of the integrand is $\frac{\delta}{d_{m}}$, with $d_{m}$ the typical size of the mesh elements (so $d_{m}=1 \mathrm{~m}$ for the self patch example). Taking a closer look at realistic values of $\frac{\delta}{d_{m}}$, two frequency ranges need to be treated. In the case of high frequencies, $d_{m}$ will be of the order of $\frac{\lambda}{10}$ (with $\lambda$ the wavelength in the background medium), while in the low frequency regime, $d_{m}$ is determined by the geometry and can be considered independent of the frequency. In the high frequency regime, with $d_{m} \approx \frac{\lambda}{10}$, we have that $\frac{\delta}{d_{m}} \approx \frac{10}{\pi c} \sqrt{\frac{\omega}{2 \mu \sigma}}$. As the frequency increases, the skin depth decreases as $\frac{1}{\sqrt{\omega}}$, but the discretisation of the triangles as $\frac{1}{\omega}$, eventually leading to a situation where our special approach is no longer required as the dimensions of the triangles become even smaller than $\delta$. However, for copper, $\frac{\delta}{d_{m}}=1$ for $\omega=$ $1.4 \cdot 10^{18} s^{-1}$ (or $f=2.2 \cdot 10^{5} \mathrm{THz}$ ), so in practice any high frequency simulation for the microwave and millimeter wave range involving copper (or other good conductors) requires the techniques we previously described. At low frequencies, we have $\frac{\delta}{d_{m}}=\frac{1}{d_{m}} \sqrt{\frac{2}{\omega \mu \sigma}}$ and the parameter depends both on the frequency and the geometry. An important aspect that has not yet been discussed before are the conditions under which it is allowed to use triangles that are considerably larger than the skin depth $\delta$. Roughly said, this is valid when the curvature of the geometry is sufficiently smooth in comparison with $\delta$. As a result, near sharp corners of a conducting object it will still be necessary to refine the mesh in order to accurately catch the electromagnetic behaviour. However, this can be done in a localised manner, without affecting the mesh of those parts that are smooth.

\section{B. Neighbour Patch}

Whilst the self patch is critical for the contribution due to the $\boldsymbol{T}$ operator of the PMCHWT formulation [10], the associated impedance integrals discretising the $\boldsymbol{K}$ operator are zero. The most common neighbour patches, namely those where the two triangles lie in the same plane, also result in a zero contribution [10]. As such, here we will consider the case of two orthogonal triangles that touch in one line (as shown in Fig. 8), which, incidentally, is also of considerable practical importance. $S_{i}$ is again defined by the vertices $(0,0,0),(1,0,0)$ and $(0,1,0)$, while $S_{j}$ has $(0,0,0),(1,0,0)$ and $(0,0,1)$ as its corners. The remaining logarithmic edge singularity in the outer integral is a well-known issue [10], but due to the focusing of our quadrature points in a small region near the common edge, relatively good and stable results can be obtained by simply applying a brute force Gaussian integration.

THE RELATIVE ERRORS $\left(\varepsilon_{r e l, 3}\right)$ FOR THE NUMERICAL EVALUATION OF THE IMPEDANCE INTEGRAL $\boldsymbol{I}_{t 3}$ IN THE CASE OF AN ORTHOGONAL NEIGHBOUR PATCH.

\begin{tabular}{|c|c|c|c|c|}
\hline $\mathrm{f}(\mathrm{Hz})$ & $\delta$ & $\varepsilon_{c u t}$ & $\left(n_{O}, n_{\phi}, n_{\rho}\right)$ & $\varepsilon_{\text {rel }, 3}$ \\
\hline $10^{4}$ & $6.4 \cdot 10^{-4}$ & $10^{-5}$ & $(210,8,33)$ & $2.3 \cdot 10^{-4}$ \\
$10^{5}$ & $2.0 \cdot 10^{-4}$ & $10^{-5}$ & $(210,8,33)$ & $3.1 \cdot 10^{-4}$ \\
$10^{6}$ & $6.4 \cdot 10^{-5}$ & $10^{-5}$ & $(210,8,33)$ & $1.1 \cdot 10^{-4}$ \\
$10^{7}$ & $2.0 \cdot 10^{-5}$ & $10^{-5}$ & $(210,8,33)$ & $2.9 \cdot 10^{-4}$ \\
$10^{8}$ & $6.4 \cdot 10^{-6}$ & $10^{-5}$ & $(210,8,33)$ & $9.9 \cdot 10^{-5}$ \\
\hline
\end{tabular}

The results, shown in Table VI, demonstrate that it is possible to obtain an accuracy that is more than enough for most applications. If a still better accuracy is required, certain approaches could be followed (e.g. [10]) to get rid of the remaining edge singularity, but that is beyond the scope of this paper.

\section{Thin Plate Triangles}

In a practical application, many of the impedance integrals (for interaction through a conductive medium) will be negligible, simply because the triangles are too distant and the kernel is highly lossy. In many cases, only the self patch, neighbour patches and point patches (when two triangles 
touch in exactly one point) contribute (the so-called singular integrals). However, one exception is that of very thin plates, with a thickness of the order of the skin depth or smaller. In that case, the interaction between the two walls through the conductive medium has an important contribution and needs to be accounted for. An important aspect regarding accuracy is that these integrals do not require the same accuracy as the self patch contribution because, due to the lossy nature of the medium, they are perturbations of the diagonal. If the self patch is known to $10^{-5}$ accuracy and the distance of the wall leads to a $10^{-3}$ drop in interaction strength, then only approximately $10^{-2}$ relative accuracy is required for the interactions through the wall. Any additional accuracy would get numerically lost in the uncertainty on the self patch. Our approach automatically takes this into account through the value of $r_{c u t}$. So, two types of relative errors will be given in the results, namely $\varepsilon_{\text {rel, } 2}=\frac{\left|I_{t 2}-I_{t 2, \text { ref }}\right|}{\left|I_{t 2, \text { ref }}\right|}$ and $\varepsilon_{r e l, 2, s}=\frac{\left|I_{t 2}-I_{t 2, r e f}\right|}{\left|I_{t 2, s p}\right|}$, with $I_{t 2, s p}$ the evaluation of the self patch integral corresponding to $S_{i}$. To make it more challenging, we will consider triangles that, while parallel (as is the case for thin walls), do not have a completely overlapping support. This creates some difficulties for the outer integral, solved by our approach. $S_{i}$ is defined by the vertices $(0,0,0),(1,0,0)$ and $(0,1,0)$ and $S_{j}$ by $(1,1, d),(0,1, d)$ and $(1,0, d)$, where $d$ is the thickness of the plate (and the distance between the triangles). The results will again focus on the accuracy of $I_{t 2}$. The results are shown in Table VII.

Table VII

THE RELATIVE ERRORS $\varepsilon_{r e l, 2}$ AND $\varepsilon_{r e l, 2, s}$ FOR THE NUMERICAL EVALUATION OF THE IMPEDANCE INTEGRAL $I_{t 2}$ IN THE CASE OF NEAR-SINGULAR PARALLEL TRIANGLES.

\begin{tabular}{|c|c|c|c|c|c|}
\hline $\mathrm{f}(\mathrm{Hz})$ & $d$ & $\varepsilon_{c u t}$ & $\left(n_{O}, n_{\phi}, n_{\rho}\right)$ & $\varepsilon_{r e l, 2}$ & $\varepsilon_{r e l, 2, s}$ \\
\hline $10^{4}$ & $10^{-5}$ & $10^{-5}$ & $(112,8,33)$ & $7.0 \cdot 10^{-6}$ & $3.5 \cdot 10^{-6}$ \\
$10^{4}$ & $10^{-4}$ & $10^{-5}$ & $(112,8,33)$ & $7.9 \cdot 10^{-6}$ & $3.4 \cdot 10^{-6}$ \\
$10^{4}$ & $10^{-3}$ & $10^{-5}$ & $(112,8,33)$ & $2.7 \cdot 10^{-5}$ & $3.0 \cdot 10^{-6}$ \\
$10^{4}$ & $10^{-2}$ & $10^{-5}$ & $(112,8,33)$ & 1 & $8.1 \cdot 10^{-8}$ \\
$10^{6}$ & $10^{-5}$ & $10^{-5}$ & $(112,8,33)$ & $1.0 \cdot 10^{-5}$ & $4.3 \cdot 10^{-6}$ \\
$10^{6}$ & $10^{-4}$ & $10^{-5}$ & $(112,8,33)$ & $4.1 \cdot 10^{-5}$ & $4.5 \cdot 10^{-6}$ \\
$10^{6}$ & $10^{-3}$ & $10^{-5}$ & $(112,8,33)$ & 1 & $8.1 \cdot 10^{-8}$ \\
$10^{8}$ & $10^{-5}$ & $10^{-5}$ & $(112,8,33)$ & $4.3 \cdot 10^{-5}$ & $4.6 \cdot 10^{-6}$ \\
$10^{8}$ & $10^{-4}$ & $10^{-5}$ & $(112,8,33)$ & $4.3 \cdot 10^{-5}$ & $8.1 \cdot 10^{-8}$ \\
\hline
\end{tabular}

The cases where $\varepsilon_{r e l, 2}=1$ are the result of $r_{c u t}$ being smaller than $d$ (meaning that the integral will be evaluated to zero). However, as shown by $\varepsilon_{r e l, 2, s} \leq 10^{-5}$, this is within our desired tolerance.

\section{NumERICAL EXAMPLE}

To illustrate the previously developed techniques, we will consider the practical case of very thin, conductive walls, which was in fact the original motivation for this work. If the wall thickness is of the order of the skin depth or smaller, the 'tunneling effect' cannot be neglected and a full-wave solution is required. In order to allow verification of the numerical result, a configuration will be chosen that allows comparison with an analytical solution. Figure 11 displays this geometry (not to scale), which consists of a hollow conductive sphere with radius $R$ and thickness $d$.

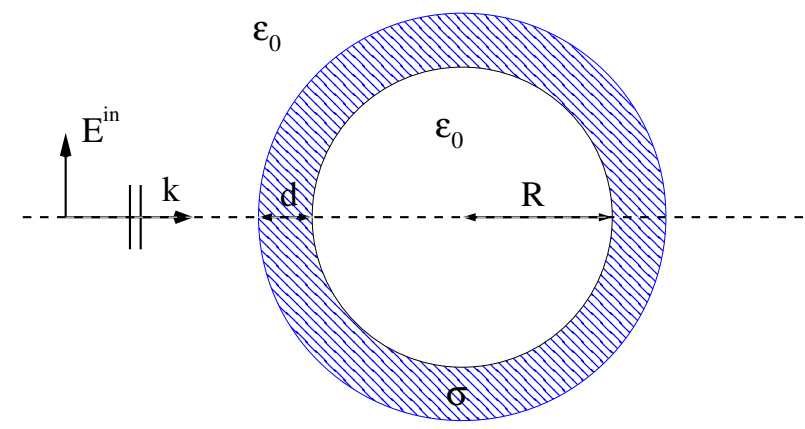

Figure 11. The geometry for the numerical example.

The parameters are chosen as follows: $R=1 \mathrm{~m}, \mathrm{~d}=10 \mu \mathrm{m}$, $\sigma=59.6 \cdot 10^{6} \mathrm{~S} \cdot \mathrm{m}^{-1}$ (copper). The incoming plane wave has a frequency of $4.77 \cdot 10^{7} \mathrm{~Hz}$ (so $k=1$ for the background medium) and is linearly polarised with $\boldsymbol{k}=(1,0,0)$ and $\boldsymbol{E}^{i n}=(0,0,1)$. The skin depth of copper at this frequency is $\delta=9.46 \mu \mathrm{m}$. The surfaces of each sphere are discretised in 584 triangles, leading to a total of 3504 unknowns. The impedance integrals were calculated with a tolerance of $10^{-5}$.

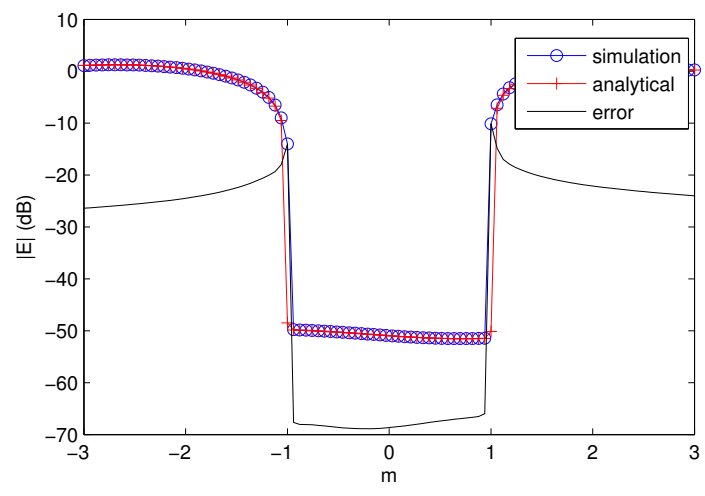

Figure 12. A comparison (between simulation and analytical result) of the electric field after scattering at a very thin conductive shell.

The results are displayed in Fig. 12, comparing the total simulated field with the analytical result obtained from the Mie series. The results are plotted along the dashed line shown in Fig. 11 (which is the $\mathrm{X}$-axis). The error is represented as $10 \log _{10}\left(\left|E_{\text {sim }}-E_{a n}\right|\right)$, which is a measure for the distance in the complex plane. As such, it compares the complex field values, taking both amplitude and phase into account. The distance between the data and the error can be interpreted as the relative accuracy of the result. This is better than $1 \%$, except close to the walls. This is due to geometrical meshing error (flat triangles are used to model a curved surface). Similar results are very difficult to obtain with a method that discretises the volume instead of the boundaries. In order to catch this behaviour it is, however, necessary to accurately evaluate the impedance integrals. An identical simulation, but using traditional Singularity Cancellation (without $r_{c u t}$ ) instead of our method, failed to converge.

In a second simulation, using the same geometry as shown in Fig. 11, we evaluate the Shield Penetration (SP) for these 
enclosures for various values of $d$. The SP, in this case, is defined as

$$
S P=20 \log _{10}\left(\frac{|\boldsymbol{E}(0,0,0)|}{\left|\boldsymbol{E}^{\text {in }}(0,0,0)\right|}\right)
$$

The results are shown in Fig. 13 for $\frac{d}{\delta}$ ranging from 0.1 to 10 , with the error defined in the same way as for Fig. 12. Clearly the simulations agree very well with the analytical solution throughout the entire domain.

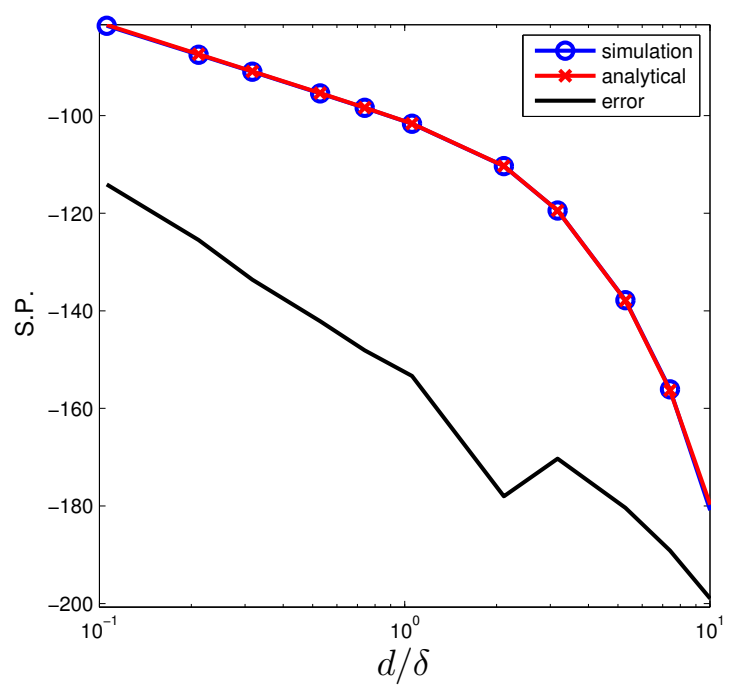

Figure 13. The Shield Penetration as a function of $d$, calculated both analytically and numerically.

Of course, the accurate modeling of the tunneling effect through a conductor is not the only application of this work. A full-wave treatment (as opposed to using, e.g., a surface impedance approximation) is necessary whenever the thickness becomes of the order of the skindepth or when the inside becomes important for other reasons, e.g. in the study of the effect of corners or of impurities within the conductor. Additionally, it provides a smooth extension of the full-wave approach for dielectrics to conductors, without requiring a sudden transition to surface impedances, possibly leading to more reliable results in the transition zone. It may also serve as a reference against which different high conductivity approaches can be evaluated. A deeper investigation of all these applications will be the subject of future work.

\section{CONCLUSION}

In this paper, the accurate and scalable evaluation of impedance integrals in a conductive medium has been treated. An error-controllable approach was proposed that is stable for the high conductivity limit, evaluating both the inner and outer integral with care. The main novelty is with regard to the use of a cut-off distance - at various places in the algorithm to more efficiently focus numerical effort. The performance of the approach was shown through a few challenging case studies (self patch, neighbour patch and near singular case) and the example of very thin conductive shells. Finally, some suggestions for application of this technique were listed.

\section{REFERENCES}

[1] D. Wilton, S. Rao, A. Glisson, D. Schaubert, O. Al-Bundak, and C. Butler, "Potential Integrals for Uniform and Linear Source Distributions on Polygonal and Polyhedral Domains," IEEE Trans. Antennas Propagation, vol. 32, no. 3, pp. 276-281, Mar. 1984.

[2] P. Ylä-Oijala and M. Taskinen, "Calculation of CFIE Impedance Matrix Elements with RWG and $n \times$ RWG Functions," IEEE Trans. Antennas Propagation, vol. 51, no. 8, pp. 1837-1846, Aug. 2003.

[3] R. Graglia, "On the Numerical Integration of the Linear Shape Functions Times the 3D-Green's Function or its Gradient on a Plane Triangle," IEEE Trans. Antennas Propagation, vol. 41, no. 10, pp. 1448-1455, Oct. 1993

[4] T. Eibert and V. Hansen, "On the Calculation of Potential Integrals for Linear Source Distributions on Triangular Domains," IEEE Trans. Antennas Propagation, vol. 43, no. 12, pp. 1499-1502, 1993.

[5] M. Khayat and D. Wilton, "Numerical Evaluation of Singular and Near-Singular Potential Integrals," IEEE Trans. Antennas Propagation, vol. 53 , no. 10 , pp. $3180-3190$, Oct. 2005.

[6] L. Rossi and P. Cullen, "On the Fully Numerical Evaluation of the Linear-Shape Function Times the 3D Green's Function on a Plane Triangle," IEEE Trans. Microwave Theory Tech., vol. 47, no. 4, pp. 398-402, Apr. 1999.

[7] R. Graglia and G. Lombardi, "Machine Precision Evaluation of Singular and Near Singular Integrals by Use of Gauss Quadrature Formulas for Rational Functiuns," IEEE Trans. Antennas Propagation, vol. 56, no. 4, pp. 981-998, Apr. 2008.

[8] J. Van Bladel, Electromagnetic Fields, ser. IEEE Press Series on Electromagnetic Wave Theory. Wiley, 2007, ISBN 978-0-471-26388-3.

[9] S. Chakraborty and V. Jandhyala, "Evaluation of Green's Function Integrals in Conducting media," IEEE Trans. Antennas Propagation, vol. 52, no. 12, pp. 3357-3363, 2004.

[10] P. Ylä-Oijala, M. Taskinen, and S. Järvenpää, "Analysis of surface integral equations in electromagnetic scattering and radiation problem," Engineering Analysis with Boundary Elements, vol. 32, no. 3, pp. 196209, Mar. 2008.

[11] S. M. Rao, D. R. Wilton, and A. W. Glisson, "Electromagnetic scattering by surfaces of arbitrary shape," IEEE Trans. Antennas Propagation, vol. AP-30, no. 3, pp. 409-418, May 1982.

[12] A. Polimeridis and J. Mosig, "Evaluation of Weakly Singular Integrals Via Generalized Cartesian Product Rules Based on the Double Exponential Formula," IEEE Trans. Antennas Propagation, vol. 58, no. 6, pp. 1980-1988, Jun. 2010.

[13] M. Mori, "Discovery of the Double Exponential Transformation and Its Developments," Publ. RIMS, Kyoto Univ., vol. 41, pp. 897-935, 2005. 